\title{
Some New Views on Teaching French with Respect to Teaching/ Learning English (Czech Experience)
}

\section{[Quelques perspectives pour une didactique du français après l'enseignement/ apprentissage de l'anglais (expérience tchèque)]}

\author{
Marie Fenclova
}

DOI: $10.18355 /$ XL.2015.08.04.2-9

\begin{abstract}
Résumé
Dans l'intervention proposée, je présenterai ce qui est actuellement à relever de l'expérience tchèque dans le domaine d'une didactique du français - deuxième langue étrangère après l'enseignement/apprentissage de l'anglais première langue étrangère. Nous soutenons l'opinion que cette approche ouvre des perspectives de la didactique intégrée des langues dont un des objectifs est le plurilinguisme de la jeune génération. Avant tout, je présenterai les résultats d'une enquête adressée aux enseignants et d'une autre enquête adressée aux enseignés concernant les représentations des deux groupes sur l'influence de l'expérience précédente d'un enseignement/apprentissage de l'anglais $1^{\text {ère }}$ langue étrangère sur un enseignement/ apprentissage suivant du français $2^{\text {ème }}$ langue étrangère. Ensuite, je montrerai une partie des erreurs les plus fréquentes que les élèves tchèques de français commettent sous l'influence de l'anglais, et dont on est en train d'établir l'inventaire provisoire. Pour finir, j'informerai de la mise en place récente d'un cours expérimental que la faculté des lettres de l'Université de Plzen offre aux étudiants de FLE, futurs enseignants de français, et dont l'objectif est de comparer le français et l'anglais et de développer une réflexion concernant les impacts positifs et négatifs pour l'enseignement de la deuxième langue étrangère.

Mots-clé

français-langue étrangère, anglais-langue étrangère, didactique intégrée des langues, transferts, stratégies
\end{abstract}

Il ne fait aucun doute que ni le français, ni l'allemand, ni une autre langue européenne ne peuvent entrer en concurrence avec l'anglais en position de première langue étrangère dans les écoles tchèques de même que dans la majorité des écoles d'Europe. Mais la primauté quantitative ne va pas forcément de pair avec la qualité de l'enseignement/apprentissage et de ses résultats. On peut gagner souvent plus facilement si l'on part de la seconde place et ce non seulement sur la piste d'athlétisme. Mais encore faut-il savoir comment procéder. Les enseignants de français en République Tchèque commencent à réfléchir aux stratégies qui pourraient changer les inconvénients en avantages. Dans la plupart des pays d'Europe, dont les politiques linguistiques introduisent obligatoirement deux langues étrangères dans les systèmes scolaires dès l'école primaire, les enseignants de français se rendent compte du fait que leurs élèves commençant à étudier le français ont déjà une expérience antérieure de l'apprentissage de l'anglais. Cette réalité évidente n'est pas suffisamment traitée du point de vue théorique et les contenus de la formation des enseignants la mentionnent très rarement. La didactique des langues vivantes devrait en tirer les conséquences, élaborer des stratégies de l'enseignement des deuxièmes langues étrangères.

Dans la situation de l'école tchèque, il est impossible de concevoir la didactique intégrée des langues étrangères sous forme d'un enseignement intégré de 
deux ou même plusieurs langues, les langues étant enseignées comme disciplines séparées. Pourtant, il est tout à fait possible et souhaitable de soutenir l'intégration de l'enseignement/apprentissage des langues accentuant le respect des didactiques des langues "suivantes » à l'égard des langues "précédentes». Les enseignants des langues «suivantes» doivent respecter l'expérience que leurs élèves ont de l'apprentissage de la langue/des langues "précédente/s ».

Dans l'intervention proposée, je veux présenter ce qui est actuellement à relever de l'expérience tchèque (plutôt modeste jusqu'ici) dans ce domaine. Avant tout, je présenterai les résultats d'une enquête adressée aux enseignants et d'une autre enquête adressée aux enseignés concernant les représentations des deux groupes sur l'influence de l'expérience précédente d'un enseignement/apprentissage de l'anglais $1^{\text {ère }}$ langue étrangère (A1LE) sur un enseignement/apprentissage suivant du français 2 ème langue étrangère (F2LE). Ensuite, je montrerai une partie des erreurs les plus fréquentes que les élèves de français commettent sous l'influence de l'anglais, et dont on est en train d'établir l'inventaire provisoire. Pour finir, j'informerai de la mise en place récente d'un cours expérimental que la faculté des lettres de l'Université de Plzen offre aux étudiants de FLE, futurs enseignants de français, et dont l'objectif est de comparer le français et l'anglais et de développer une réflexion concernant les impacts positifs et négatifs pour l'enseignement de la deuxième langue étrangère.

Je travaille depuis plusieurs années à l' Université de Bohême de l'Ouest où un des programmes d'études au niveau de licence est consacré aux langues française et anglaise avec orientations professionnelle et commerciale, et la formation suivante, au niveau du master, qui a pour but la formation des enseignants de français (FLE et FOS). Nos étudiants en master de FLE ont alors une bonne connaissance des deux langues et nous pouvons nous reporter aux résultats d'une recherche que nous réalisons depuis 2007 parmi les enseignants et les étudiants/élèves de français. Les résultats de 2007 ont été complétés en 2009 et 2010 de sorte qu'on a reçu 960 questionnaires remplis par les élèves et 45 autres questionnaires remplis par les professeurs.

Pour commencer, je présenterai quelques résultats de ces enquêtes dont une partie a été publiée en 2008 (Fenclová; Dlesková 2008). Il s'en suit que :

- $\quad$ Presque $98 \%$ de débutants en français dans les écoles tchèques ont déjà une expérience antérieure de l'apprentissage de l'anglais.

- $\quad$ Plus de la moitié (57\%) des élèves pense que l'apprentissage précédant de l'anglais les aide lors de l'apprentissage du français. Leurs réponses signalent que l'éventuel aspect positif de l'impact dépend de la qualité de l'enseignant de l'une et de l'autre langue. Les élèves expliquent leurs réponses positives par la ressemblance du vocabulaire $(87 \%)$ et, moins souvent, la ressemblance de certains phénomènes grammaticaux (7\%). Il est surtout à retenir que $10 \%$ d'élèves remarquent aussi l'avantage de l'expérience précédente du contact avec une langue étrangère à l'égard de leur apprentissage d'une nouvelle langue étrangère. C'est-à-dire, ces élèves se rendent compte des avantages des aptitudes cognitives formées préalablement au cours de l'apprentissage de l'anglais.

- Pour ce qui est des enseignants, il est symptomatique qu'ils remarquent plutôt les conséquences des transferts négatifs de l'anglais en français. Moins souvent, ils se rendent compte des possibilités de profiter du transfert positif et des compétences métacognitives et métalinguistiques que les élèves ont acquises au cours de l'apprentissage précédent de l'A1LE. C'est sur ce point que la formation des enseignants devrait se focaliser. 
Dans le questionnaire, nous voulions relever aussi l'inventaire des transferts de l'anglais en français que les enseignants tchèques remarquent.

Dans nos questionnaires, nous nous sommes intéressées en premier lieu aux transferts positifs. 93\% d'enseignants constatent une influence positive dans l'appropriation du lexique, $66 \%$ d'enseignants la remarquent aussi en grammaire.

\section{Les transferts positifs}

\section{Le lexique}

Pour ce qui est du lexique, le répertoire des expressions de même origine est important dans les deux langues, qu'il s'agisse de latinismes, d'emprunts similaires à d'autres langues ou de mots d'origine française en anglais et de mots d'origine anglaise en français. Les enseignants remarquent les avantages qui s'en suivent pour l'enseignement. Inutile d'énumérer de nombreux exemples de ressemblances évidentes, telles que table - la table, family - la famille

\section{La grammaire}

Pour ce qui est de la grammaire, les enseignants croient remarquer quelques traits de transfert positif pour des phénomènes comme :

- l'ordre des mots fixe dans la phrase énonciative (ce qui n'est pas le cas en tchèque)

- l'existence de pronoms conjoints et indépendants en français de même qu'en anglais : my - mine / mon - le mien (en tchèque on dispose seulement d'une forme pour les deux cas)

- la distinction de la nombrabilité/innombrabilité des substantifs qui a, dans les deux langues, des conséquences grammaticales : en anglais le choix entre muchmanylfew - little, en français le choix de l'article partitif ou autre (Cette distinction en tchèque ne joue aucun rôle.)

- l'existence et la distribution des articles indéfinis et définis (We have a cat. - The cat is old./ Nous avons un chat. - Le chat est vieux.) (Le tchèque n'a pas d'article, il utilise dans les deux cas seulement le nom.)

- les structures de la voix passive des verbes

- les règles de concordance des temps qui se ressemblent dans les deux langues

- les types similaires des phrases hypothétiques pour l'expression de la condition

Tous ces cas témoignent du fait que l'impact du transfert positif de l'anglais première langue étrangère sur le français deuxième langue étrangère se manifeste du fait de l'absence de cette analogie entre la langue maternelle (le tchèque) et les deux langues étrangères (l'anglais et le français). Par exemple, en présentant les structures de la voix passive des verbes dans la phrase française, il est utile de s'appuyer sur la structure similaire de la phrase anglaise : English is spoken all over the world. - Le français est parlé dans beaucoup de pays. En effet cette analogie peut éviter l'interférence de la structure tchèque Francouzsky se mluví v mnoha zemích dont la traduction littérale Le français se parle dans beaucoup de pays n'est pas appropriée.

\section{Autres transferts positifs}

Les enseignants ont remarqué aussi la tendance chez les étudiants à chercher des comparaisons dans les questions de civilisation et de culture. Par exemple, ayant parlé d'halloween en anglais, ils veulent savoir si quelque chose de similaire existe aussi en France et dans d'autres pays francophones. 
En général, de bonnes connaissances en anglais provoquent parfois la curiosité des élèves de savoir comment cela se dit en français... S'ils ont appris une certaine conjonction par exemple en anglais, ils veulent la connaître aussi en français, etc.

\section{Les transferts négatifs}

Il est naturel que les transferts négatifs (les interférences) attirent plus l'attention des professeurs (et oui, les enseignants sont toujours fascinés par les fautes !). Dans nos questionnaires, nous trouvons beaucoup d'exemples de toutes sortes. Le plus souvent il s'agit de la prononciation, de la grammaire, de faux-amis lexicaux (ce qui concerne aussi l'orthographe) et d'autres.

\section{La prononciation}

Dans la prononciation, il s'agit des exemples suivants :

- Les transferts des séquences sonores des mots apparentés automatisés par l'élève en anglais : music - musique, natural - naturel, cousin - cousin, excuse - excuse. C'est très évident pour les mots en - ion, - ation: information, transformation, decision, condition... (n'oubliant pas l'accent qui doit déménager en français sur la dernière syllabe). Pourtant, il ne s'agit pas uniquement de mots apparentés, c'est aussi le cas de la prononciation de jusqu'à en [dzaska] et d'autres.

- La prononciation en français de la consonne finale muette : complet sous l'influence du complete [kompli:t] anglais, le président sous l'influence du president anglais. Cela concerne très souvent aussi le $\underline{\mathrm{s}}$ du pluriel.

- L'omission de la nasalité (surtout au début de mots) : importent, intention...

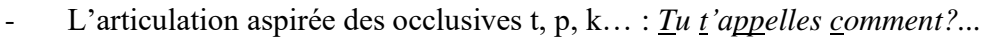

- La vibrante $\mathrm{R}$ est réalisée (pas seulement par les débutants) à la manière anglaise ou plutôt américaine (Prague, Paris, Aveiro).

Il nous semble que c'est justement dans ce domaine que l'élaboration d'un système d'exercices visant l'élimination des interférences anglaises est un but réaliste, réalisable assez rapidement.

\section{Le lexique}

Quant au lexique, les enseignants ont noté beaucoup d'exemples intéressants d'interférences entre l'anglais et le français, entre autres du type de fauxamis. Les étudiants utilisent le car au lieu de la voiture, le palace au lieu du palais. Ils tiennent le sens du verbe travailler pour identique au verbe anglais travel, confondent le sens de la journée et celui de journey. Vu la fonction du people en anglais, ils utilisent le peuple au lieu des gens. L'influence de l'anglais se manifeste dans les locutions du type Je suis dix pour l'âge, ou Je suis un étudiant utilisant l'article.

\section{La grammaire}

En ce qui concerne l'influence non souhaitable dans le domaine de la grammaire, les enseignants remarquent le plus souvent les fautes suivantes :

- la place de l'adjectif avant le nom (quoique ce problème soit lié aussi et avant tout à la langue maternelle)

- l'omission des caractéristiques grammaticales de l'épithète en français qui doit, à la différence de l'anglais, s'accorder avec son substantif (un repas chaud-des boissons chaudes) 
- les représentations erronées sur les correspondances entre les deux systèmes de temps verbaux (l'imparfait n'a pas en français les mêmes fonctions que le « past continuous » en anglais).

- L'omission de la conjonction relative que dans les structures comme: Invitons chaque personne que nous connaissons! / Let's invite everyone we know!

Dans cet ordre d'idées, il est assez intéressant de constater que les enseignants de français se plaignent de l'omission des articles comme d'un fait très répandu chez ceux qui ont étudié déjà l'anglais. Nous trouvons cela plutôt surprenant, car nous attendrions plutôt un mécanisme de transfert positif. Mais il est évident que l'on ne peut exploiter l'effet des transferts positifs qu'à condition que les compétences des élèves en anglais, sur lesquelles nous voulons axer notre présentation du français, soient assez solides, ce qui n'est pas toujours le fait en pratique scolaire.

\section{L'orthographe}

Pour ce qui est de l'orthographe, les enseignants enregistrent par exemple l'absence de l'accent circonflexe sous l'influence des anciens emprunts anglais au français comme le dîner (dinner), la forêt (forest). Ils notent aussi des exemples d'alternances métathétiques, c'est-à-dire de l'inversion de lettres dans l'orthographe du novembre français et november anglais, la lettre et letter. On note parfois l'élision dans le pronom personnel devant le verbe j'parle sous l'influence de I'm speaking (mais dans ce dernier cas on peut supposer aussi une interférence intralinguale, c'està-dire une fausse analogie agissant à l'intérieur du français $: j$ 'ai $\rightarrow j$ 'suis).

\section{Les stratégies}

Enfin, nous avons posé aux enseignants plusieurs questions concernant les stratégies de l'enseignement. On a constaté que seulement 3 sur nos 45 informateurs ne se réfèrent jamais aux connaissances de l'anglais de leurs étudiants, tandis que 27 informateurs le font de temps en temps et 15 même souvent.

Nous avons recueilli beaucoup d'exemples, mais le plus souvent on note la sémantisation d' expressions abstraites : curieux - curious, similaire - similar, la nécessité - necessity, la promotion - promotion...

Puis nous avons enregistré certaines structures grammaticales au cours de la présentation desquelles les enseignants renvoient aux similarités avec l'anglais :

- le rôle des articles

- l'ordre de mots

- la concordance des temps ( ?)

- les locutions there is/are - il y a

- les structures conditionnelles

En ce qui concerne les stratégies d'apprentissage, c'est-à-dire les habitudes que les étudiants avaient acquises lors de l'apprentissage de l'anglais, les enseignants rappellent plusieurs faits. Toutefois il faut constater que ces aptitudes ne sont pas réellement propres à tous les élèves ayant déjà étudié une/des langue/s étrangère/s :

- l'habitude d'imiter la prononciation de la langue égrangère

- $\quad$ l'habitude de l'approche globale en compréhension de texte (il ne faut pas connaître tous les mots pour comprendre) 
- $\quad$ le fait de savoir que l'on ne doit pas «traduire » littéralement

- le courage de s'exprimer à l'aide d'une langue autre que la langue maternelle

- la volonté élémentaire de communiquer (y compris avec des gestes et des mimiques)

- l'habitude d'utiliser le dictionnaire (les élèves savent déjà que les synonymes trouvés dans un dictionnaire ne sont pas pleinement interchangeables dans n'importe quel contexte).

- la familiarisation avec quelques types d'exercices (textes lacunaires, vrai faux...)

- $\quad$ l'acceptation de la nécessité d'un travail régulier et systématique (sic).

En général, les enseignants expriment le désir de disposer d'un support introduction didactique à l'enseignement du F2LE, c'est-à-dire après l'anglais.

Tout cela a confirmé notre hypothèse qu'il est souhaitable de soutenir dans la didactique tchèque du français :

1) les recherches visant l'élaboration d'un support pour les enseignants du français 2LE après l'anglais $1 \mathrm{LE}$;

2) l'introduction de la problématique dans les programmes de formation des enseignants de français.

Les deux buts sont en train (ou plutôt au début) de leur réalisation. Le premier fait l'objet de la thèse d'un de mes doctorants. Pour ce qui est de l'autre, à l'Université de Plzen, nous avons récemment mis en place un cours expérimental que la faculté des lettres offre aux étudiants du FLE, futurs enseignants de français. Son objectif est de comparer le français et l'anglais et de développer la réflexion des étudiants - futurs enseignants - concernant les impacts positifs et négatifs pour l'enseignement de la deuxième langue étrangère. L'enseignant choisit les sujets basés sur la comparaison des systèmes de l'anglais, du français et du tchèque.

Logiquement il existe 5 variantes de similarités/dissimilarités entre les phénomènes linguistiques dans ces trois langues (français - anglais - tchèque) :

1) $\mathbf{F}=\mathbf{A}=\mathbf{T}$ (le fait linguistique correspond dans les trois langues)

Certains adjectifs ne peuvent avoir ni comparatif ni superlatif, exprimant eux-mêmes la supériorité (perfect-parfait-perfektní, unique - unique - unikátní)

2) $\mathbf{F} \neq \mathbf{A} \neq \mathbf{T}$ (le fait linguistique n'est similaire ni entre le français et l'anglais, ni entre l'anglais et le tchèque, ni entre le tchèque et le français)

Les formes de la négation verbale (I don't now - Je ne sais pas-Nevím)

Le genre des substantifs : en anglais pas de distinction formelle (the/a), en français genre masculin et genre féminin (le, la/un, une), en tchèque les genres masculin, féminin et neutre (implicite au substantif lui-même)

L'utilisation des prépositions différentes après les adjectifs

responsible for responsable de zodpovédný za

surprised by ou at surpris par ou de prekvapený (čím)

pleased with content $\underline{\mathrm{de}}$ spokojený $\underline{\mathrm{s}}$

3) $\mathbf{F}=\mathbf{T} \neq \mathbf{A}$ (la situation en français correspond à la situation en tchèque, en anglais c'est différent)

système de tutoiement et de vouvoiement,

pas de majuscules aux noms de jours, de mois, de saisons 
4) $\mathbf{F} \neq \mathbf{A}=\mathbf{T}$ (la situation correspond entre le tchèque et l'anglais, mais ne correspond pas au français)

la marque du pluriel pour les noms propres,

le même mot pour l'exclamation et pour l'interrogation (how kind you are!/

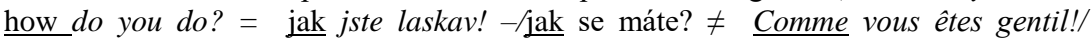
Comment allez-vous?)

5) $\mathbf{F}=\mathbf{A} \neq \mathbf{T}$ (Il y a la similarité entre le français et l'anglais, mais en tchèque c'est différent)

La nécessité d'exprimer le pronom sujet dans la phrase en anglais de même qu'en français, en différence du tchèque (I study - $\underline{\mathrm{J}}$ étudei - Studuji) (en tchèque la conjugaison est basée sur la flexion)

La nécessité de distinguer les pronoms conjoints et indépendants en anglais de même qu'en français en différence du tchèque : my - mine / mon-le mien/ můj.

Dans notre séminaire, les étudiants complètent le répertoire d'après les catégories précédentes et essaient de proposer des activités et exercices en fonction des donnés recueillies. Il s'agit surtout de la valorisation des transferts positifs de l'anglais en français et de l'élimination des transferts négatifs.

\section{Conclusion}

Les propositions des stratégies basées sur l'appuit de l'enseignement/ apprentissage du français-première langue étrangère sur les savoirs et savoirs-faire acquis au cours de l'enseignement/apprentissage de l'anglais-première langue étrangère apportent les premières preuves d'un impact positif, néanmoins la condition en est un niveau solide de la maîtrise de l'anglais ce qui n'est pas toujours le cas dans la réalité scolaire.

\section{Références bibliographiques}

BAILLY, S. et al. 2009. L'anglais langue d'appui pour l'apprentissage du français langue etrangere. In: Forlot, G. (ed.) L'anglais et le plurilinguisme : Pour une didactique des contacts et des passerelles linguistiques. Paris: L'Harmattan, 2009. ISBN 978-2-296-10859-2.

FORLOT, G. 2009. L'anglais et le plurilinguisme : Pour une didactique des contacts et des passerelles linguistiques. Paris: L'Harmattan. ISBN 978-2-296-10859-2.

BONO, M. 2007. La comparaison L2-L3, un tremplin vers 1'acquisition trilingue. Birbeck Studies. In Applied Linguistics. 2007b, vol. 2, pp. 22-41. ISSN 1754-5366.

CANDERLIER, M. - KERVRAN, M. - REMY-THOMAS, F. 2003. Une approche plurielle des langues a l'ecole primaire. Construire de nouvelles competences preparatoires a la traduction. Le français d'aujourd'hui, 2003/3 ( $\left.\mathrm{n}^{\circ} 142\right)$, pp. 55-67. http://www.cairn.info/revue-le-francais-aujourd-hui-2003-3-page-55.htm

DUVERGER, J. 2000. De l'interet des approches contrastives. Entretien avec Claire Blanche-Benveniste. Le Français dans le Monde. 2000, N. 311, pp. 95-97. ISSN 0015-9395.

FENCLOVA, M. - DLESKOVA, H. 2008. L'apprentissage de l'anglais/ l'apprentissage du français: ce qu'en pensent les enseignants/ce qu'en pensent les enseignes. In: Le français - deuxieme langue etrangere. La didactique integree des langues etrangeres. Plzen: Zapadoceska univerzita v Plzni. ISBN: 978-80-7043-721-6 FENCLOVA, M. - HOROVA, H. 2008. Le français - deuxieme langue etrangere La didactique integree des langues etrangeres. Plzen: ZCU. pp. 19 - 30. ISBN 978-807043-721-6. 
HOROVA, H. 2008. A la recherche des strategies pour le français deuxieme langue etrangere. In: FENCLOVA, M. - HOROVA, H. (eds.) Le français - deuxieme langue etrangere - La didactique integree des langues etrangeres. Plzen: ZCU. pp. 19 - 30. Pp. 12 - 18. ISBN 978-80-7043-721-6.

KERVRAN, M. 2011. Didactique convergente du langage et des langues a l'ecole primaire: le role de la memoire didactique. Revue française de pedagogie. Recherches en education, 2011/2 ( $\left.\mathrm{n}^{\circ} 175\right)$, pp. 89-98. ISSN 0556-7807.

KOCOUREK, R. 2001. L'anglais et le français: rapports entre deux langues. In: Comparaisons, contrastes, correspondances: le français et l'anglais en terminologie et en langue de spécialité. Halifax: Université Dalhousie. ALFA, vol. 9, pp. 5-18.

ROBERT, J.-M. 2009. Manieres d'apprendre. Pour des strategies d'apprentissage differenciees. chap. 2.4. L'anglais comme langue passerelle, pp. 85-95. Paris : Hachette. ISBN 2011556805.

VERCOLLIER, A. - VERCOLLIER, C. - BOURLIER, K. 2004. Difficultes expliquees du français for English Speakers. Paris: CLE International. ISBN 978 209033701-3.

MEISEL, J. 1983. Transfer as a second language strategy. Language and communication. 1983, N. 3, pp. 11-46. ISSN 1460-6984.

ROBERT, J. M. 2008. L'anglais comme langue proche du français ? Ela. Études de linguistique appliquée. 2008, N. 149. ISSN 1965-0477. [online Available www.cairn.info/revue-ela-2008-1-p-9.htm]

ROBERT, J.-M. 2009. Manieres d'apprendre. Pour des strategies d'apprentissage differenciees. Chap. 2.4. L'anglais comme langue passerelle, pp. 85-95. Paris : Hachette. ISBN 2011556805

VAN LIER, H. 2004. L'entente cordiale. Complementarite du français et de l'anglais. Le français dans le monde, No 336, 2004, pp. 38-41.

VÍT, R. 2013. Didakticke vyuyiti znalosti, dovednosti, ucebnich strategii a navyku osvojenych pri uceni anglictiny (L2) pro vyuku francouzstiny (L3). Disertacni prace, PedF UK v Praze. [online Available https://is.cuni.cz/webapps/zzp/detail/93620/]

VÍT, R. 2015. Mezijazykovy transfer pri uceni druheho ciziho jazyka. Cizi jazyky, 57/5. 57/5, pp. 11-18. ISSN 1210-0811

Words: 3252

Characters: 21714 (12,06 standard pages)

Doc. PhDr. Marie Fenclová, CSc.

Boheme University of Pilsen

Faculty of Letters

Riegrova 11, 30614 Plzen̆

Czech Republic

fenclova@kro.zcu.cz 